\title{
Multiflow Feasibility: an Annotated Tableau
}

\author{
Guyslain Naves, András Sebő
}

Laboratoire G-SCOP, CNRS, INPG, UJF, 46 avenue Félix Viallet 38031 Grenoble Cedex, France $^{\star}$

Summary. We provide a tableau of 189 entries and some annotations presenting the computational complexity of integer multiflow feasibility problems; 21 entries remain open. The tableau is followed by an introduction to the field, providing more problems, reproving some results with new insights, simple proofs, or slight sharpenings motivated by the tableau, paying particular attention to planar (di)graphs with terminals on the boundary. Last, the key-theorems and key-problems of the tableau are listed.

Keywords: disjoint paths, multiflows, planar graphs, acyclic digraphs.

\begin{tabular}{|c|c|c|c|c|c|c|c|c|c|c|c|}
\hline & \multicolumn{3}{|c|}{ directed } & \multicolumn{3}{|c|}{ directed acyclic } & \multicolumn{3}{|c|}{ undirected } \\
\hline & & & \multicolumn{2}{|c|}{ arc-disjoint } & \multirow{2}{*}{$\begin{array}{l}\text { vertex- } \\
\text { disjoint }\end{array}$} & \multicolumn{2}{|c|}{ arc-disjoint } & \multirow{2}{*}{$\begin{array}{l}\text { vertex- } \\
\text { disjoint }\end{array}$} & \multicolumn{2}{|c|}{ edge-disjoint } & \multirow{2}{*}{$\begin{array}{l}\text { vertex- } \\
\text { disjoint }\end{array}$} \\
\hline $\mathrm{G}$ & $\mathrm{E}(\mathrm{H})$ & $\mathrm{r}$ & gen & Eulerian & & gen & Eulerian & & gen & Eulerian & \\
\hline \multirow{9}{*}{ gen } & \multirow{2}{*}{$\operatorname{arb}$} & bin & $\mathrm{NPC}$ & $\mathrm{NPC}$ & \multirow{2}{*}{ NPC } & $\mathrm{NPC}$ & $\mathrm{NPC}$ & \multirow{2}{*}{$\mathrm{NPC}$} & $\mathrm{NPC}$ & $\mathrm{NPC}$ & \multirow{2}{*}{ NPC $^{4}$} \\
\hline & & un & $\mathrm{NPC}$ & NPC & & NPC & $\mathrm{NPC}$ & & $\mathrm{NPC}$ & $\mathrm{NPC}$ & \\
\hline & \multirow{3}{*}{ fix } & bin & NPC & NPC & \multirow{3}{*}{ NPC } & NPC & NPC & \multirow{3}{*}{$\mathbf{P}^{2}$} & NPC & NPC & \multirow{3}{*}{$\mathbf{P}^{12}$} \\
\hline & & un & $\mathrm{NPC}$ & NPC & & $\mathrm{NPC}$ & $\mathbf{N P C}^{19}$ & & $\mathrm{NPC}$ & $\mathrm{NPC}$ & \\
\hline & & fix & $\mathrm{NPC}$ & $? ? ?^{3}$ & & $\mathrm{P}$ & $\mathrm{P}$ & & $\mathrm{P}$ & $\mathrm{P}$ & \\
\hline & \multirow{4}{*}{2} & bin & NPC & $\mathbf{P}^{10}$ & \multirow{4}{*}{$\mathbf{N P C}^{2}$} & NPC & $\mathrm{P}$ & \multirow{4}{*}{$\mathrm{P}$} & NPC & $\mathbf{P}^{13}$ & \multirow{4}{*}{$\mathrm{P}$} \\
\hline & & un & $\mathrm{NPC}$ & $\mathrm{P}$ & & $\mathbf{N P C}^{1}$ & $\mathrm{P}$ & & NPC $^{1}$ & $\mathrm{P}$ & \\
\hline & & fix & NPC & $\mathrm{P}$ & & $\mathrm{P}$ & $\mathrm{P}$ & & $\mathrm{P}$ & $\mathrm{P}$ & \\
\hline & & 2 & $\mathrm{NPC}^{2}$ & $\mathrm{P}$ & & $\mathrm{P}$ & $\mathrm{P}$ & & $\mathrm{P}$ & $\mathrm{P}$ & \\
\hline \multirow{9}{*}{ plan } & \multirow{2}{*}{ arb } & bin & $\mathrm{NPC}$ & $\mathrm{NPC}$ & \multirow{2}{*}{ NPC } & NPC & NPC & \multirow{2}{*}{$\mathrm{NPC}$} & NPC & NPC & \multirow{2}{*}{$\mathrm{NPC}^{7}$} \\
\hline & & un & NPC & NPC & & $\mathbf{N P C}^{19}$ & $\mathrm{NPC}^{20}$ & & $\mathrm{NPC}^{5}$ & NPC & \\
\hline & \multirow{3}{*}{ fix } & bin & $\mathrm{NPC}$ & $? ? ?$ & & NPC & $? ? ?$ & & NPC & $? ? ?$ & \\
\hline & & un & NPC & $? ? ?$ & $\mathbf{P}^{14}$ & \begin{tabular}{|l|}
$\mathbf{N P C}^{16}$ \\
\end{tabular} & $? ? ?$ & $\mathrm{P}$ & $\mathbf{N P C}^{16}$ & $? ? ?$ & $\mathbf{P}^{15}$ \\
\hline & & fix & $? ? ?$ & $? ? ?$ & & $\mathrm{P}$ & $\mathrm{P}$ & & $\mathrm{P}$ & $\mathrm{P}$ & \\
\hline & & bin & NPC & $\mathrm{P}$ & & NPC & $\mathrm{P}$ & & NPC & $\mathrm{P}$ & \\
\hline & 2 & un & NPC $^{9}$ & $\mathrm{P}$ & $\mathrm{P}$ & $\mathbf{N P C}^{11}$ & $\mathrm{P}$ & $\mathrm{P}$ & $\mathbf{N P C}^{11}$ & $\mathrm{P}$ & $\mathrm{P}$ \\
\hline & & fix & $? ? ?$ & $\mathrm{P}$ & & $\mathrm{P}$ & $\mathrm{P}$ & & $\mathrm{P}$ & $\mathrm{P}$ & \\
\hline & & 2 & $? ? ?$ & $\mathrm{P}$ & & $\mathrm{P}$ & $\mathrm{P}$ & & $\mathrm{P}$ & $\mathrm{P}$ & \\
\hline & arb & bin & NPC & $? ? ?$ & $\mathrm{NPC}$ & $\mathbf{P}^{6}$ & $\mathrm{P}$ & 19 & NPC & $\mathbf{P}^{18}$ & $\mathrm{C}^{8}$ \\
\hline & dev & un & NPC & $? ? ?$ & $1 \mathrm{NT}$ & $\mathrm{P}$ & $\mathrm{P}$ & 10 & $\mathrm{NPC}^{8}$ & $\mathrm{P}$ & $1+0$ \\
\hline & & bin & $\mathrm{NPC}$ & $? ? ?$ & & $\mathrm{P}$ & $\mathrm{P}$ & & $\mathbf{P}^{17}$ & $\mathrm{P}$ & \\
\hline$G+H$ & fix & un & NPC & $? ? ?$ & $\mathrm{P}$ & $\mathrm{P}$ & $\mathrm{P}$ & $\mathrm{P}$ & $\mathrm{P}$ & $\mathrm{P}$ & $\mathrm{P}$ \\
\hline plan & & fix & $? ? ?$ & $? ? ?$ & & $\mathrm{P}$ & $\mathrm{P}$ & & $\mathrm{P}$ & $\mathrm{P}$ & \\
\hline & & bin & $\mathrm{NPC}$ & $\mathrm{P}$ & & $\mathrm{P}$ & $\mathrm{P}$ & & $\mathrm{P}$ & $\mathrm{P}$ & \\
\hline & & un & $\mathbf{N P C}^{11}$ & $\mathrm{P}$ & $\mathrm{P}$ & $\mathrm{P}$ & $\mathrm{P}$ & $\mathrm{P}$ & $\mathrm{P}$ & $\mathrm{P}$ & $P$ \\
\hline & 2 & fix & $? ? ?$ & $\mathrm{P}$ & & $\mathrm{P}$ & $\mathrm{P}$ & & $\mathrm{P}$ & $\mathrm{P}$ & \\
\hline & & 2 & ??? & $\mathrm{P}$ & & $\mathrm{P}$ & $\mathrm{P}$ & & $\mathrm{P}$ & $\mathrm{P}$ & \\
\hline
\end{tabular}

* Supported by the Marie Curie Training Network "ADONET" of the European community 


\subsection{Introduction}

Finding a set of (vertex- or edge-) disjoint paths in (directed or undirected) graphs between given pairs of terminals is one of the most ancestral and most studied themes of graph theory, with important applications such as routing problems of VLSI design [10]. The scope of the methods and objectives is large and spread in time: Menger's theorems or more generally network flows are among the first consistent results of combinatorial optimization [32], whereas finding edge- or vertex-disjoint paths between a given (fixed) number of terminal pairs in polynomial time is a deep pure graph theory result [26]. A multiflow is the packing of one of the simplest objects in graphs: paths. At the same time it is an integer point in a naturally defined polyhedral cone. The field has been developed in parallel with the tools of optimization, polyhedral combinatorics and graph theory. Some branches were and are still the subject of extensive studies both by the inner stimulus of the theory and the request of the applications.

Nevertheless, while the variety of the possibilities is endless, some interesting questions may not even have been realized. It is even more frustrating that at the borderlines of existing theories there are forgotten problems that have no reason to be missing. The idea of making this tableau arose when the authors got confused in varying the defining parameters of problems: which are the combinations of the parameters that lead to polynomial solvable, NP-hard or unsolved problems. A careful focus on these showed that some of the interesting combinations have not yet been studied at all.

For some kind of disjoint paths problems there exist classifications, for instance in Schrijver's book [32] or that of Korte and Vygen [11], or in survey papers of the collection [10], like [7].

A (integer) multiflow - first informally - is just a multiset of paths satisfying request and capacity constraints. The difference is not essential comparing to disjoint paths problems as far as assertions about them are concerned, however, there may be a difference in the algorithmic point of view: in multiflow problems there are numbers associated to edges or vertices, and in a solution - called a multiflow - a multiplicity is given with every path, and we want the algorithms to deal with the multiplicities in a clever way. From this viewpoint multiflows are points of a cone.

In this note we wish to focus merely on the existence of multiflows with particular attention to different natural special cases involving planarity, the number of demands, the way the capacities are given, and Eulericity. We restrict ourselves to feasibility, that is the existence of disjoint paths between all pairs of given terminal pairs. Another important direction is multiflow maximization (or maximum number of disjoint paths) that we do not treat here, since we would then have to cover yet other vast theories handled by quite different methods, and where approximation algorithms and APX-completeness should also be accounted. Exact methods concerning this subject,

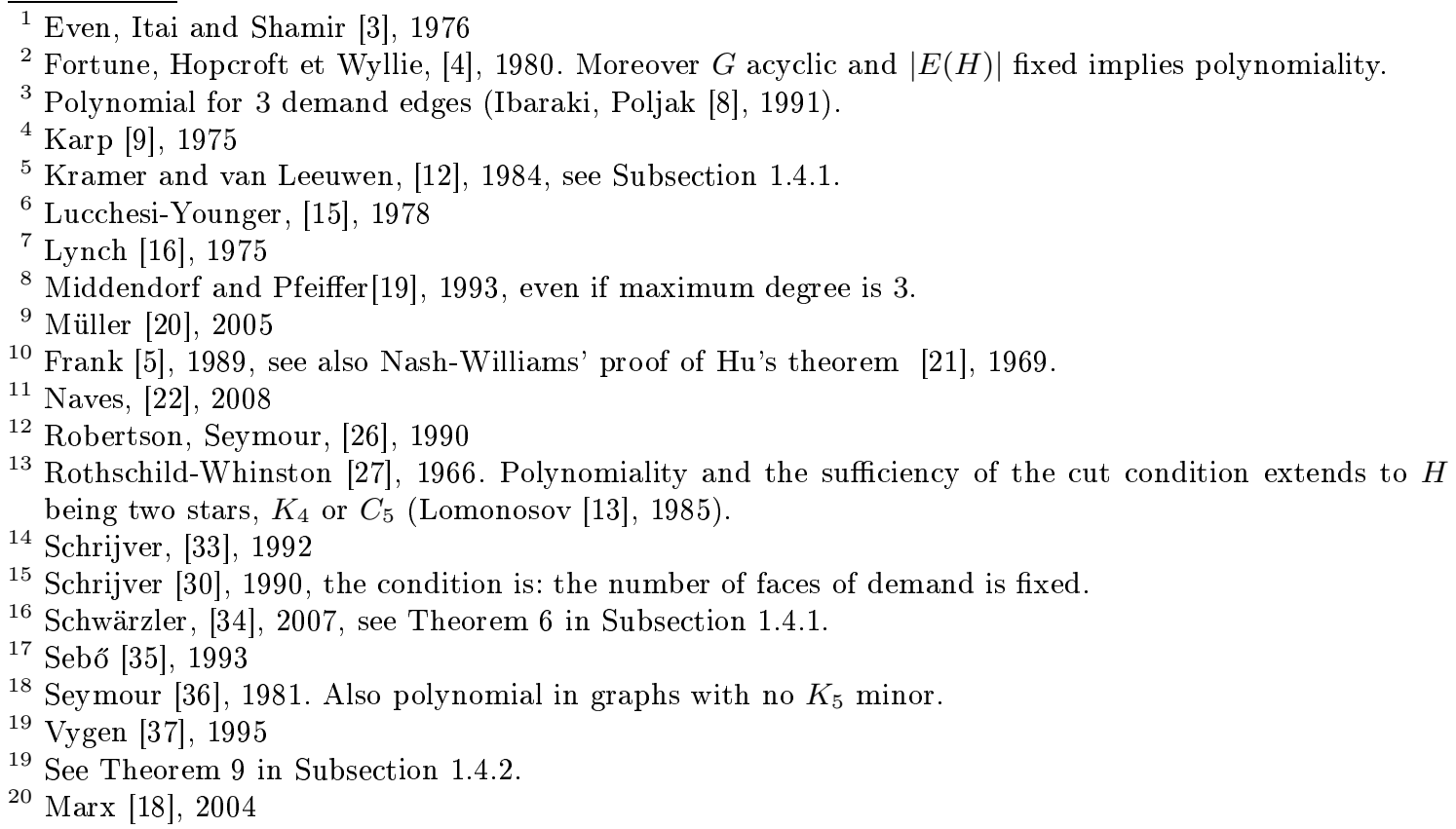


such as Mader's theorem are treated in the above mentioned books, and some other aspects like approximability are surveyed in the work of C. Bentz, M.-C. Costa, L. Létocart and F. Roupin [2] and in the thesis work of C. Bentz [1].

There are also many derivates of the problem. We had to be selective for keeping enough attention for the problems that occur in the most basic circle in the focus of our magnifying lens.

The main "product" of our work is the tableau on the first page. In the tableau we tried to concentrate on a small number of natural row (column) heads that can be nontrivially matched by most columns (rows) so as to cover most of the relevant problems. More problems (like the Okamura-Seymour circle of questions) will be discussed in the text without charging the tableau.

In Section 1.2, we provide the first explanations concerning the tableau, and the most important notations. Section 1.3 is a short introduction to the basic methods concerning multiflows.

The traces of the unsolved problems of the tableau lead to the particular graphs treated in details in Section 1.4: planar (di)graphs. The undirected planar case (Subsection 1.4.1) seems to be almost the same as the acyclic planar case (Subsection 1.4.2), the arguments for one can be repeated for the other, but we do not see any formal reduction between the two sets of instances.

When we started our work, more than one third of these problems were open. While we were working, two fundamental problems have been solved, one of them stimulated by this tableau. Schwärzler's result [34] started the row, solving Problem 56 in [32]: disjoint paths in planar graphs when all terminal pairs are on the boundary of the infinite face. This proof opened new hopes of reaching longstanding open problems and simplifiying complicated proofs:

In Schwärzler's proof there are three natural classes of pairs of terminals, so it is not difficult to prove NP-hardness if the number of terminals is restricted to 3 , and we will show the reduction below. With essential new ideas the first author has then shown [22] that 3 can be decreased to 2 , thus filling in new squares of the tableau, and solving a problem of Müller [20] about planar graphs in general, and replacing Müller's quite involved proof for the directed version. We hope the tableau will provide similar stimulation for the 21 still unfilled squares.

To make this guided tour more pleasant, we occasionnally provide some new viewpoints or variants of results, simple proofs or remarks on the way.

\subsection{Basic Notation and Annotation}

We hope the tableau is making clear the limits of different complexity behaviors (polynomiality and NP-completeness) and of the open cases. This also requires the realization of some connections. We introduce now the most important definitions, notations and conventions for a correct interpretation of the tableau.

Let $G=(V, E)$ be a graph, for the moment we allow $G$ to be undirected or directed, and $n:=|V|$. Let us call a function $c: E \rightarrow \mathbb{N}$ be a capacity function, and $H=(T, D), T \subseteq V$ a demand graph with a request function $r: D \rightarrow \mathbb{N}$. Then the multiflow problem is to find a multiset $\mathcal{C}$ of cycles in $G+H$ verifying the following condition:

- for each cycle $C \in \mathcal{C},|C \cap D|=1$,

- for each $d \in D$, there are exactly $r(d)$ cycles in $\mathcal{C}$ that contain $d$,

- for each $e \in E$, there are at most $c(d)$ cycles in $\mathcal{C}$ that contain $e$.

The integrality of the multiplicities of cycles is supposed. In the rare cases when it is not, we will speak about fractional multiflows.

If $r$ and $c$ are both 1 everywhere we speak about edge-disjoint (or in digraphs arc-disjoint) paths problems.

By analogy, we could define, both in directed and undirected graphs, vertex-capacitated multiflow problems, vertex-disjoint paths problems by putting capacities and demands on vertices, and by repeating the three conditions above by replacing circuits $C$ by their vertices, $H$ simply by a vertex-set $D \subseteq V$, and $E$ by $V \backslash D$.

If we still want to keep a demand graph $H$, we can, by putting a new vertex $d_{e}$ in the middle of each edge $(\operatorname{arc}) e=t u$ of $H$, and letting $r\left(d_{e}\right):=c(t)=c(u)$. 
The choices for the rows and columns of the tableau are of course partly a matter of taste. However we tried to distinguish the different problems along some basic parameters that the community cares about:

The first three columns of the tableau concern

- restrictions of $G$ and $H$ : general, $G$ planar or $G+H$ planar

- restrictions on the cardinality of $E(H)$ : arbitrary, fix or 2

- restrictions on the size of $r$ and the way it is given: "bin" means binary encoding, "un" unary encoding, that is, the size of the input is measured by the sum of the given numbers instead of the sum of their logarithms; "fix" means that $\sum_{e \in E(H)} r(e)$ is bounded.

Even though the restrictions never concern $c$ directly, it is naturally affected: if $r$ is unary, we can suppose without loss of generality that $c$ is also. (The sum of $c$ on all edges can be supposed to be at most $n$ times the sum of $r$.)

The distinction between "bin" and "un" is the same as the usual distinction between pseudopolynomiality and strong NP-complete: for instance when $H$ has two edges, unary encoding is equivalent to putting as many parallel copies of the edges in $H$ as the demand, and similarly for the capacities; so the unary problem with $|E(H)|=2$ is the same as the edge-disjoint paths problem with two parallel classes of demand edges, and is NP-complete. However, the "fix" version is polynomially solvable by Robertson and Seymour [26].

The same holds for all edge-disjoint paths problems: multiflows with "unary" encoding are nothing more than edge-disjoint paths problems with maybe restricted $H$ (like in the example) and several parallel demand edges.

The "bin" case could be essentially more difficult than the unary. Indeed, in a binary encoding we are not allowed to replace the capacities by parallel edges, since a polynomial algorithm must then work in time which is polynomial in the input size. In this case the input size is the sum of the logarithms of the capacities. Surprisingly, this does not drastically change the complexity of the problems: in our tableau the "bin" cases have exactly the same complexity as the "un" ones. An explanation of this lies probably in the classical Ford Fulkerson theory of network flows: the paths through each demand edge obey the same rules as ordinary network flows, the difficult problem is to split the problem between the different terminal pairs.

Another kind of relation occurs between "fix" and "un" or "bin" if $G+H$ is planar and $H$ has a bounded number of edges, that is, $|E(H)|$ is "fix": then $r$ "fix" may again be settled by [26], but this does not solve the "un" or "bin" case, and turns out not to be the best solution for "fix" either. Indeed, "bin" can also be solved in polynomial time, by applying Lenstra's "cheaper" integer programming algorithm [35].

Thus in the edge-disjoint case "bin" and "un" can be thought of as being the same, and allowing an arbitrary number of parallel classes of demand edges; "fix" $|E(H)|$ restricts the number of parallel classes of demand edges, and "fix" in the $r$ column the total number of demand edges. The latter of course implies the former.

The situation is somewhat more complicated in the vertex-disjoint case. For vertex-capacitated multiflows the unary case has to be distinguished from vertex-disjoint paths if $G$ is restricted for instance to planar graphs. The replication of vertices (replacing the parallel edges of the reduction of multiflows to edge-disjoint paths), does not keep for instance the planarity of $G$.

Besides edge- or arc- and vertex-disjoint paths problems we also distinguish the same problems under the Eulerian condition:

We distinguish between $G+H(\mathrm{r}+\mathrm{c})$ Eulerian or not (gen): if $G, H$ are undirected, $(G, H, r, c)$ is called Eulerian if for each $v \in V$,

$$
\sum_{e \in \delta_{G}(v)} c(e)+\sum_{d \in \delta_{H}(v)} r(d) \text { is even }
$$

and if $G, H$ are digraphs, then the Eulerian property means for each $v \in V$,

$$
\sum_{e \in \delta_{G}^{+}(v)} c(e)+\sum_{d \in \delta_{H}^{+}(v)} r(d)=\sum_{e \in \delta_{G}^{-}(v)} c(e)+\sum_{d \in \delta_{H}^{-}(v)} r(d) .
$$


The four-tuple $(G, H, r, c)$ will not necessarily be always explicitly mentioned - most of these parameters are fixed by the context.

In this paper the main focus is the edge-disjoint paths problem and multiflows. The columns concerning vertex-disjoint paths are present for comparison and all suppose that the request and capacity functions are both 1 everywhere, that is, we are looking only at vertex-disjoint paths problems, and none of the new problems that are raised:

Problem 1 Fill in additional columns of the tableau for vertex-capacitated problems, where the vertex requests and capacities are not supposed to be 1 , but are encoded with a unary or binary encoding.

Note that the unary case cannot always be reduced to the vertex-disjoint paths problem in the same class of graphs.

Paths and cycles will always be simple, and the terms are used both in directed and undirected graphs. Our notations will be usual; $\delta(X)(X \subseteq V)$ denotes the set of edges with exactly one endpoint if $X$, and $X, V \backslash X$ are the shores of this cut.

In several cases we will also have particular notes for the case when $H$ has three edges. Another particular case of $H$ is when it is a star: then the problem can be reduced to a flow problem, and thus the problems are polynomially solvable for any $G$.

\subsection{Basic facts}

\subsubsection{Well-known reductions}

We recall some well-known reductions between the different cases that are fully exploited in the tableau.

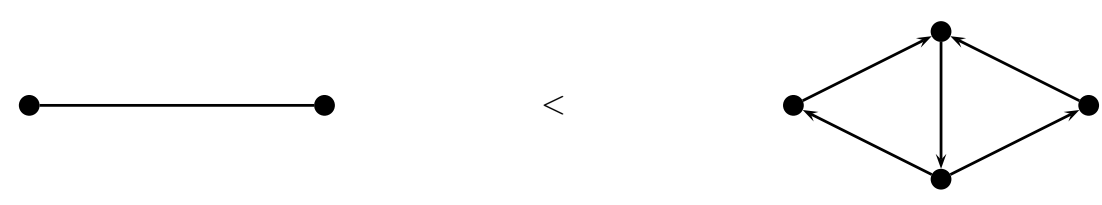

Fig. 1.1. The undirected case is reducible to the directed case, using this gadget. Only one path can use these five arcs, either from left to right or from right to left.

The undirected case can be reduced to the directed one by replacing each edge by the gadget depicted in figure 1.1. Note that this reduction preserves the planarity of $G$ and $G+H$, but does not preserve the Euler property, and the resulting graph is not acyclic.

The edge-disjoint case is reducible to the vertex-disjoint one by taking the line-graph. This operation does not keep the planarity of $G$. It works in the directed case as well with the appropriate definition of the line graph (stars of vertices become complete bipartite graphs by joining all the vertices corresponding to incoming edges to all those corresponding to outgoing edges).

In theedge-disjoint case, it is possible to reduce every graph with max-degree greater than 4 to a graph with degrees at most 4 , by using the gadget of picture 1.2 , which also keeps planarity. (The capacities must be 1.) In the particular case when $G+H$ is planar, it was remarked in [19] that in the uncapacitated case the maximum degree can be restricted to 3 , thus the edge-disjoint paths problem is reducible to the vertex-disjoint paths problem. This allows to confirm the negative complexity of some vertex-disjoint paths problems but one has to proceed carefully, since $|E(H)|$ increases. 

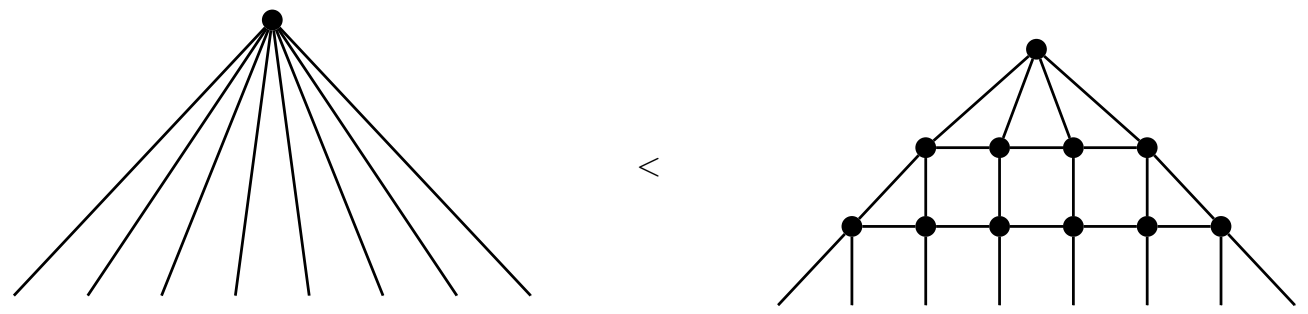

Fig. 1.2. In the edge-disjoint case, this gadget reduces the degrees of vertices to 4 .

The following lemma was proposed by Vygen [37]. It proves the equivalence between the acyclic arc-disjoint paths problem in Eulerian digraphs and the edge-disjoint paths problem (in Eulerian graphs).

Vygen's lemma: Let $(G, H)$ be an instance of the arc-disjoint paths problem, assume $G+H$ is Eulerian and that $G$ is acyclic. Let $\left(G^{\prime}, H^{\prime}\right)$ be the instance of the edge-disjoint paths problem obtained by neglecting the orientation of $G$ and $H$. Then there exists a solution for the arc-disjoint paths problem in $(G, H)$ if and only if there exists a solution for the edge-disjoint paths problem $\left(G^{\prime}, H^{\prime}\right)$.

More exactly, it is proved that the solutions of these two problems can be transformed to one another by neglecting the orientation or conversely by orienting the edges depending on the orientation of $G$.

\subsubsection{Conditions}

A solution of the (fractional or integer) multiflow problem can be seen as the problem of deciding the existence of an (integer) point in a given particular polytope. Using an idea of Lomonosov [13] we provide a compact formulation of a 'lifting' of this polytope, that is, we provide a polytope with a polynomial number of constraints in the input size whose projection is the multiflow polytope. The conditions for multiflow feasibility can be seen as valid inequalities for this polytope.

Let $G=(V, E), c, H=(T, D), r$ be an instance of the multiflow problem. Paths and cycles, directed or undirected will always supposed to be simple, that is, contain each edge at most once. Let $\mathcal{C}$ be the set of the cycles of $G+H$ that contain exactly one edge from $H$. Then the solutions of the disjoint paths problem are in bijection with the integer solutions of the following linear program:

$$
\begin{array}{r}
\sum_{d \in C, C \in \mathcal{C}} x_{C}=r(d) \quad(d \in D) \\
\sum_{e \in C, C \in \mathcal{C}} x_{C} \leq c(e) \quad(e \in E) \\
x_{C} \geq 0 \quad(C \in \mathcal{C})
\end{array}
$$

Equations 1.3 and 1.4 define the (fractional) multiflow polytope. A multiflow is an integer point of this polytope.

The nonemptyness of this polytope can be characterized by Farkas's lemma:

Theorem 1 (Japanese theorem) [24],[32] The existence of a multiflow is equivalent to the distance criterion

$$
\text { For all } w: E \longrightarrow \mathbb{R}_{+}, \sum_{(u, v) \in D} r(u, v) \times d_{G, w}(u, v) \leq \sum_{e \in E} w(e)
$$

We can obtain an easy consequence by taking, for each cut $C$, the weight function $w: E \rightarrow\{0,1\}$ defined by $w(e)=1$ iff $e \in \delta_{H}(C)$. This gives the following condition called the cut condition:

$$
\text { for all } C \subset V,\left|\delta_{G}(C)\right| \geq\left|\delta_{H}(C)\right|
$$


A cut is called tight if equality holds in (1.7) for this cut. Another interesting necessary condition for the existence of integer multiflows when $G+H$ is not Eulerian is that the union of any number of tight cuts (as edge-sets) must not contain an odd cut. (The reason is that each edge of such an odd cut is used by a multiflow, that is, the disjoint circuits of a multiflow partition the cut. However, each class of this partition is even. Intersecting the shore of (any number of) cuts, if $X$ is the intersection, $\delta(X)$ will be contained in the union of the cuts.)

Therefore if the intersected shores define all tight cuts, the intersection must define an even cut (if an integer multiflow exists). We do not know many papers where this is exploited; the nicest example is probably Frank [6], which uses this condition for two tight cuts.

We show now that the condition 1.7 of the Japanese theorem can be handled as a linear program of polynomial size, and at the same time we show the polarity between metrics and multiflows.

A function $\mu: V \times V \longrightarrow \mathbb{Z}_{+}$is called a metric on $V$, if it satisfies the triangle inequality

$$
\mu(x, y)+\mu(y, z)-\mu(x, z) \geq 0 \text {, for all } x, y, z \in V .
$$

The integrality requirement is superfluous, we only suppose it for confort. Let us denote $t(x, y, z) \in\{0,1,-1\}^{V \times V}$ which takes the value 1 on $(x, y)$ and $(y, z),-1$ on $(x, z)$, and 0 on all the other ordered pairs. Denote $T$ the matrix whose columns are the vectors of the form $t(x, y, z)$ for all ordered triples $(x, y, z),(x, y, z \in V)$. The metrics are then the solutions of the systems of inequalities $y T \geq 0$. The following nice observation is due to Lomonosov [13]:

Let $P:=\left(v_{1}, \ldots, v_{k}\right)$ be a path, and $v_{P} \in\{0,1,-1\}^{V \times V}, v_{P}(x, y)=1$ if $x=v_{i}, y=v_{i+1}$ for all $i=1, \ldots, k-1$, and $v_{P}\left(v_{k} v_{1}\right)=-1$. Then

$$
v_{P}=\sum_{i=2}^{k-1} t\left(v_{1}, v_{i}, v_{i+1}\right),
$$

and therefore for $c \in R^{V \times V}$, the solutions of the system of linear inequalities $T x \leq c, x \geq 0$ are in one-to-one correspondance with the (fractional) multiflows in the graph $G=(V,\{e \in V \times V$ : $c(e)>0\}$, with capacity $c$, and demand graph $H, u v \in E(H)$ if and only if $c(u v)<0$, and then $r(u v):=-c(u v)$. (For undirected multiflow problems we use only one of $u v$ and $v u$ ). Integer solutions of this system correspond to (integer) multiflows. Note that $T$ has a polynomial number of entries, immediately implying polynomial solvability of fractional multiflow problems and the interested reader may find it useful to rewrite the Farkas' Lemma for this somewhat different system of inequalities.

\subsection{Planar Graphs}

In this section we state and sometimes improve or reprove results about the complexity of multiflows in planar graphs. The results concerning undirected graphs can often be translated to acyclic digraphs.

\subsubsection{Undirected Graphs}

This subsection updates the complexity of the planar edge-disjoint paths problem.

Two of the first important results of the subject are that of Lynch [16] stating that the vertexdisjoint paths problem is NP-complete in planar graphs, and the sharpening of Kramer and van Leeuwen [12] to grid graphs. The latter result has the advantage of being easy to manipulate to prove NP-completeness of variants of the problem such as edge-disjoint paths problems: the authors themselves note that the problem remains NP-complete if common edges are still not allowed, but common vertices may occur provided the two paths "cross" in those. Raghavan [25][Lemma 2.1] notes that the edge-disjoint problem is also NP-complete with "their reduction". This is right noting that the last part of Kramer and van Leeuwen's proof has to be - slightly and in a straightforward way - modified in order to get NP-completeness of the general planar edge-disjoint paths problem.

In planar routing problems the terminals are often on the boundary of the infinite face. We want to explore the complexity of problems satisfying this condition.

We start with a new proof of the classical Okamura-Seymour theorem providing a polynomial algorithm for planar Eulerian graphs with all terminals on the outer face. We continue by sketching 
Schwärzler's proof of the NP-completeness of the non-Eulerian case, and show a slight extension where in addition the number of demand edges can be restricted to three. Finally, we sketch the more involved new ideas that allowed the first author to achieve the last possible step and prove that the same holds for two demand edges.

Theorem 2 (Okamura, Seymour [23]) Let $G=(V, E)$ be a planar graph and $H=(T, D), T \subseteq$ $V$ where the vertices of $T$ are on the outer boundary of the embedding of $G$. Let $r: D \rightarrow \mathbb{N}$ and $c: E \rightarrow \mathbb{N}$ be weight functions, and suppose that $r+c$ is Eulerian. Then the cut condition is sufficient for the existence of a multiflow for $(G, H)$.

We first reformulate this theorem as a statement on metric packings, and provide a proof combining a technique of Schrijver for proving distance packing theorems [32] with ideas in [14] for decomposing distance functions, and new ideas capturing the essence of Lins' theorem: in a critical situation saturated by a technique of [32], guided by the role of the "oppositeness relation" in [14] but without using the related polyhedral statements - we decompose our graph into cuts. Schrijver applies the dual of this oppositeness relation to prove Lins' theorem in the context of an inductive proof. Despite these similarities, the use of the previous results remains implicit in the proof below, and our present proof is self-contained, fully combinatorial, and hopefully generalizable. Since it seems to provide some insight, we want to communicate it for possible future use.

The theorem is equivalent to a theorem on metric packings, see Corollary 74.2a in [32], proved there using the Okamura-Seymour theorem. Here we will prove this form directly. The advantage of this method may be to provide some insight of how the metrics guide the direction the (dual) paths take.

Let us call a circuit $C \subseteq E(G)$ rigid, if for any two, $a$ and $b$ of its vertices, one of the $(a, b)$-paths on the circuit is a geodesic in $G$. (The facial structure of the cone of metrics implies that the only way to write the distance function of a graph as the sum of metrics is using cuts intersecting rigid cycles with 0 or two opposite edges [14]. This statement did guide our proof without using it.)

We prove the following reformulation of the Okamura-Seymour theorem:

Theorem 3 Let $G=(V, E)$ be a planar graph with only rigid faces, with all faces being 4-cycles except the infinite face, and where in addition any set of two successive edges of a face are together contained in a geodesic with both endpoints in the boundary $C$ of the infinite face. Then the graph $(E, \Omega)$ on the edges of $G$, where $\Omega:=\{e f: e, f \in E$, $e$ is opposite to $f$ on some face $\}$ is a graph that has $|C| / 2$ components, where each component is a path joining two opposite edges of $C$.

The conditions imply, of course, that $G$ is bipartite. Before the proof let us sketch the reduction of the Okamura-Seymour theorem (Theorem 2) to this, which consists of simple and standard steps.

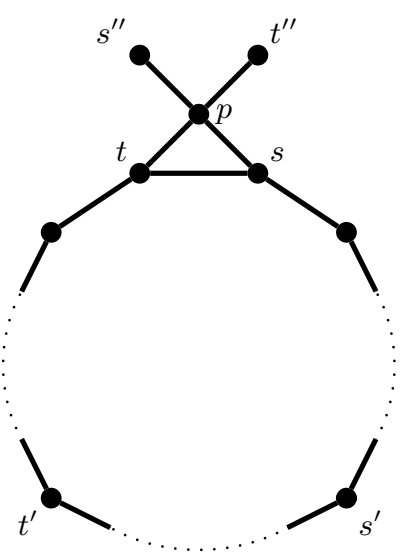

Fig. 1.3. In Okamura-Seymour's theorem, we can assume that terminals of each demand pair are diametrally opposed: add vertices $p, s^{\prime \prime}$ and $t^{\prime \prime}$ and the edges joining them as indicated on the figure, whenever $s, t, t^{\prime}, s^{\prime}$ occur in this "wrong" order along the boundary of the outer face. Replace the demand edges $s s^{\prime}, t t^{\prime}$ by $s^{\prime} s^{\prime \prime}$ and $t^{\prime} t^{\prime \prime}$. 
1. Reduce the Okamura-Seymour theorem to the case when the terminal pairs $D:=\left\{s_{1} t_{1}, \ldots, s_{k} t_{k}\right\}$ follow one another in the order $s_{1}, \ldots, s_{k}, t_{1}, \ldots, t_{k}$ on $C$, see Figure 1.3. Reduce then to the 2-vertex-connected case without changing the order of the terminals.

2. Add a new vertex $x_{0}$, place it to the infinite face and join it with all the terminal vertices. Delete each vertex of degree 2 , by merging its two edges.

3. Take the planar dual of the obtained graph.

4. Add the gadget of Figure 1.4 to all faces that are not 4-cycles, until all faces are 4-cycles.

5. Identify the opposite vertices of 4-cycles if they are not contained on a geodesic with both endpoints in $C$.
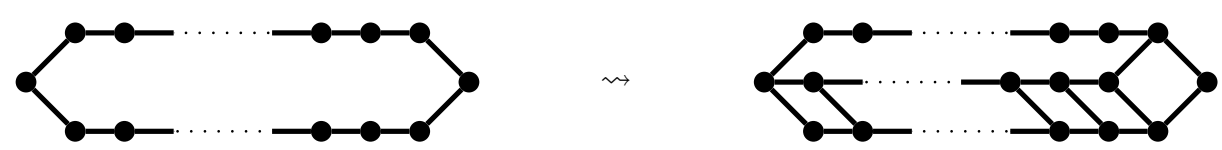

Fig. 1.4. Reducing the boundaries of faces to 4, without changing the distances, in poly-time.

It is easy to see that the cut condition implies that after applying these procedures the conditions of Theorem 3 are satisfied. The theorem then implies by dualization a set of edge-disjoint paths for the original problem, and the proof is algorithmic, straightforwardly providing a polynomial algorithm.

If $P$ is a path and $x, y$ are two of its vertices, $P(x, y)$ denotes the subpath of $P$ from $x$ to $y$.

Proof.

Claim 1: The graph $(E, \Omega)$ is the disjoint union of cycles and of $|C| / 2$ paths with both endpoints on $C$.

Indeed, in the graph $(E, \Omega)$ every edge of $C$ is of degree 1 , and any other $e \in E$ has degree 2.

Claim 2: For any cycle $D \subseteq E$ in $G$ and $a, b \in V(D)$ such that both paths $A$ and $B$ between $a$ and $b$ on $D$ are shortest paths in $G$, each component of $(E, \Omega)$ is a path that has one end in $A$, and another in $B$.
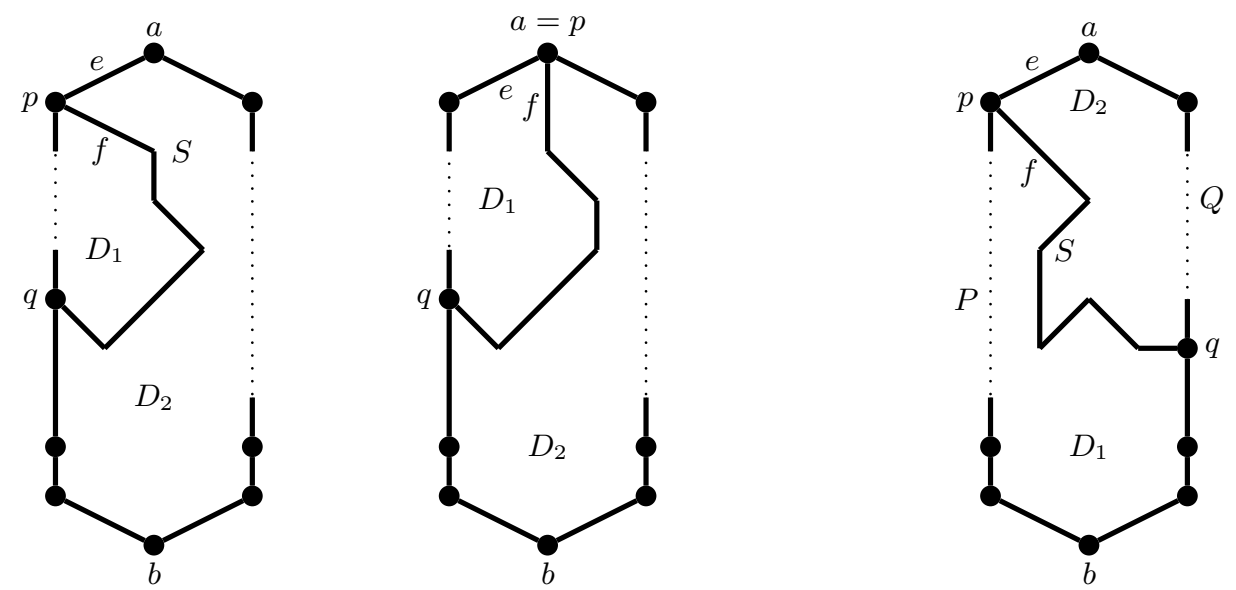

Fig. 1.5. Depending on the position of $q$, on the same $(a, b)$-path as $p$ or not, we apply the induction hypothesis to $D_{1}, p, q$ and $D_{2}, a, b$ on the two first drawings (case 1), or $D_{1}, p, b$ and $D_{2}, a, q$ on the third one (case 2), for which the condition on the distances still holds.

Indeed, if every edge of $D$ incident to $a$ is followed by a boundary edge of $D$, then $D$ is a face of $G$, and the statement is evident. Otherwise there exist two edges $e, f$ (Figure 1.5) such that

(i) $e$ and $f$ are incident edges of a face -let their common point be $p$. 
(ii) $e$ is incident to $a$.

(iii) The interior of $f$ is contained inside $D$, that is, in the open disk bounded by $D$.

By the condition there exists a geodesic path $S$ containing $e$ and $f$ and with extremities on $C$. Then starting on $S$ from $a$ on $e$ and then $f$ and continuing, let $q$ be the next vertex of $D$ (by (iii) and Jordan's theorem $q$ exists) on $S$. The subpath $S(p, q)$ divides $D$ into two cycles $D_{1}$ and $D_{2}$ that intersect in $S(p, q)$ (see the Figure). Since the subpath of a geodesic is also a geodesic, both $S(a, q)$ and $S(p, q)$ are a geodesics.

Denote $H, H_{1}, H_{2}$ the subgraph of $(E, \Omega)$ induced by $D, D_{1}, D_{2}$ and the edges inside. Informally, these are the subgraphs describing oppositeness within $D, D_{1}$ or $D_{2}$. (In $H$ the edges of $S(p, q)$ form a vertex-cut-set which, together with the two components of $H-S(p, q)$ induces the subgraphs $H_{1}$ and $H_{2}$.) Clearly, like in Claim 1 , the components of $H, H_{1}$ and $H_{2}$ are paths, and there are respectively $|D| / 2,\left|D_{1}\right| / 2,\left|D_{2}\right| / 2$ such paths.

Case 1: One of the two $(a, b)$-paths of $D$ is disjoint from $S(p, q)$.

Then applying the induction hypothesis to $D_{1}$ and $D_{2}$ (see left drawings of Figure 1.5) and merging the components of $H_{1}$ and $H_{2}$, we get the statement for $D$. (Each edge of $S(p, q)$ is the endpoint of a component in both of the graphs $H_{1}$ and $H_{2}$, and these pairs of components can be merged.)

Case 2: Both $(a, b)$-paths of $D$ meet $S(p, q)$.

Then $a p$ is the first edge of one of the two $(a, b)$-paths $P$ of $D$, and $q$ is on the other $(a, b)$-path $Q$. (Figure 1.5 right.)

Both $S(a, q)$ and $Q(a, q)$ are geodesics, and the induction hypothesis can be applied to $D_{2}$ with these geodesics. By induction we get paths of $(E, \Omega)$ one of which, $S_{2, e}$ connects the edge $e=a p$ to an edge of $Q(a, q)$, and the others, $S_{2, h}(h \in S(p, q)$ each connect edges of $S(p, q)$ to edges of $Q(a, q)$ (except the end of $S_{2, e}$ different from $e$ ). These are the components of $\mathrm{H}_{2}$.

As a side-product $|S(a, q)|=|Q(a, q)|$, and therefore

$$
|S(p, q) \cup Q(q, b)|=|Q(a, q)|-1+Q(q, b)|=| P(p, b) \mid,
$$

whence $S(p, q) \cup Q(q, b)$ is also a geodesic and the induction hypothesis can be applied to $D_{1}$ as well, and with the geodesics $S(p, q) \cup Q(q, b)$ and $P(p, b)$. So by induction, the components of $H_{1}$ are the paths $S_{1, h}\left(h \in S(p, q)\right.$ connecting $S(p, q)$ to a subset of $P(p, b)$, and $S_{1, h}(h \in Q(q, b))$ to another subset. Let $\mathcal{S}:=\left\{S_{1, h} \cup S_{2, h}: h \in S(p, q)\right\}$. Now clearly, the set

$$
\left\{S_{2, e}\right\} \cup \mathcal{S} \cup\left\{S_{1, h}: h \in Q(q, b)\right\}
$$

is the set of components of $H$, and connects each edge of $P(a, b)$ to an edge of $Q(a, b)$, finishing the proof of Claim 2.

Now applying Claim 2 to $D:=C$ and all the $|C| / 2$ pairs of geodesics each of which (bi)partitions $C$, we get that each component of $H$ connects two edges that do not lie in the same class of any of these bipartitions. It follows that the components of $H$ join opposite edges of $C$.

Frank proved that the problem is still polynomially solvable when only the inner vertices of $G$ verify the Eulerian condition:

Theorem 4 (Frank) Let $G=(V, E)$ be a planar graph and $H=(T, D),(T \subset V)$, where the vertices of $T$ are on the outer boundary of the embedding of $G$; let $r: D \rightarrow \mathbb{N}$ and $c: E \rightarrow \mathbb{N}$ be weight functions, and suppose that for each vertex $v$ not contained in the outer boundary of $G$, $\sum_{e \in \delta(v)} c(e)$ is even. Then the edge-disjoint paths problem can be solved in polynomial time.

However, the Euler property cannot be completely removed:

Theorem 5 (Schwärzler) The edge-disjoint paths problem when $G$ is planar and the terminals lie on the outer boundary of $G$ is NP-complete.

Schwärzler's gadget can be completed to reduce the number of demand edges to 3 : 
Theorem 6 The multiflow problem when $G$ is planar, $|E(H)|=3$ and the terminals lie on the outer boundary of $G$ is NP-complete.

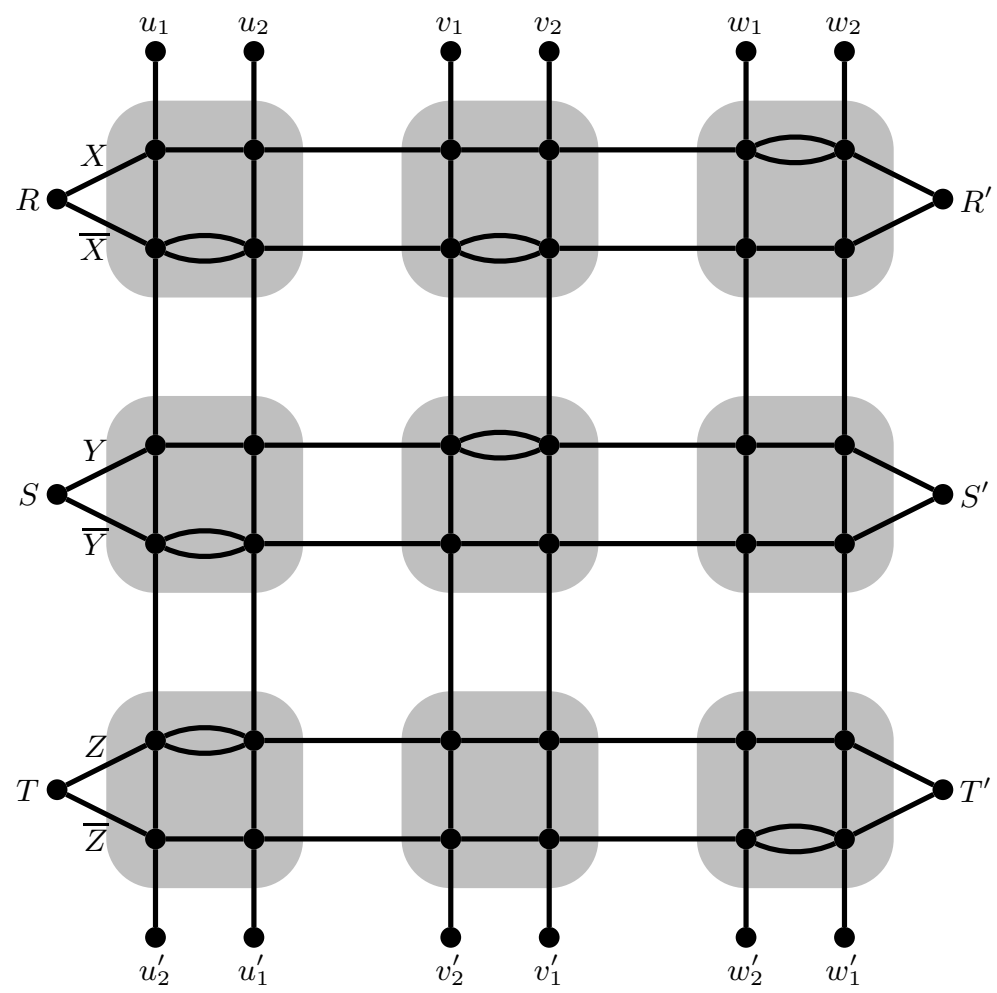

Fig. 1.6. An example of the reduction, from the formula $(X \vee Y \vee \neg Z) \wedge(X \vee \neg Y) \wedge(\neg X \vee Z)$. The drawn graph is the supply graph, the demand edges join vertices with their primes.

Proof. (Sketch) We sketch Schwärzler's proof, rearranged and completed by a reduction to three parallel classes of demand edges with a linear number of demands altogether. The reduction is from Satisfiability. From a formula given in conjunctive normal form, a grid is built with as many columns and rows as there are clauses and variables respectively. There are two lines in each column and in each row, paths in the graph, but because of the placement of the terminals, these will not be paths in a solution, see Figure 1.6. The extremities of the demand edges are labeled vertices and their primes.

In a solution two paths will join the two demand edges of each column, and one the demand edge of each row. The latter (horizontal) path of each row will be obliged to be one of the two horizontal lines of the row, and this choice corresponds to choosing a truth value for the corresponding variable: choosing the upper path means that TRUE is assigned to it, and the lower path means that FALSE is the assigned value.

The two paths of each column are forced by the order of their terminal vertices to exchange their lines. This exchange encodes the fact that each clause must be satisfied. Such an exchange is possible through two parallel horizontal "column-switch" edges. In each square of the grid the two parallel column-switch edges are placed in the upper or lower line or neither depending on whether a variable, its negation or neither are present in the corresponding clause.

By considering tight cuts, Schwärzler proves that the horizontal paths do not use any vertical edges. This is the way of forcing a horizontal path to stay in the same row and not to change lines, corresponding to a choice of truth value. Then, vertical paths can cross only through free column-switch parallel edges, making the choice of a true variable which is positive in the clause or a false one which is negated.

The number of parallel classes of demand edges can be reduced to 3 by introducing one parallel class for each "type" of demand edge: first introduce two new terminals and one demand edge for 
all the horizontal paths, this does not cause any difficulty; then construct the two parallel classes of demand edges for the vertical paths, one for paths switching from left to right, and one for those switching from right to left, with one demand edge per path, and a gadget making possible for these paths to cross in a planar way (Figure 1.7). The demand graph is reduced then to only three sets of parallel edges - one horizontal, and two vertical ones. To check that this operation does not change the problem, note:

The tight cuts represented by the dashed lines force all starting points of both lines of all columns to be contained in different vertical paths. It can be shown by induction from left to right that these paths are rooted like in the previous part of the proof.

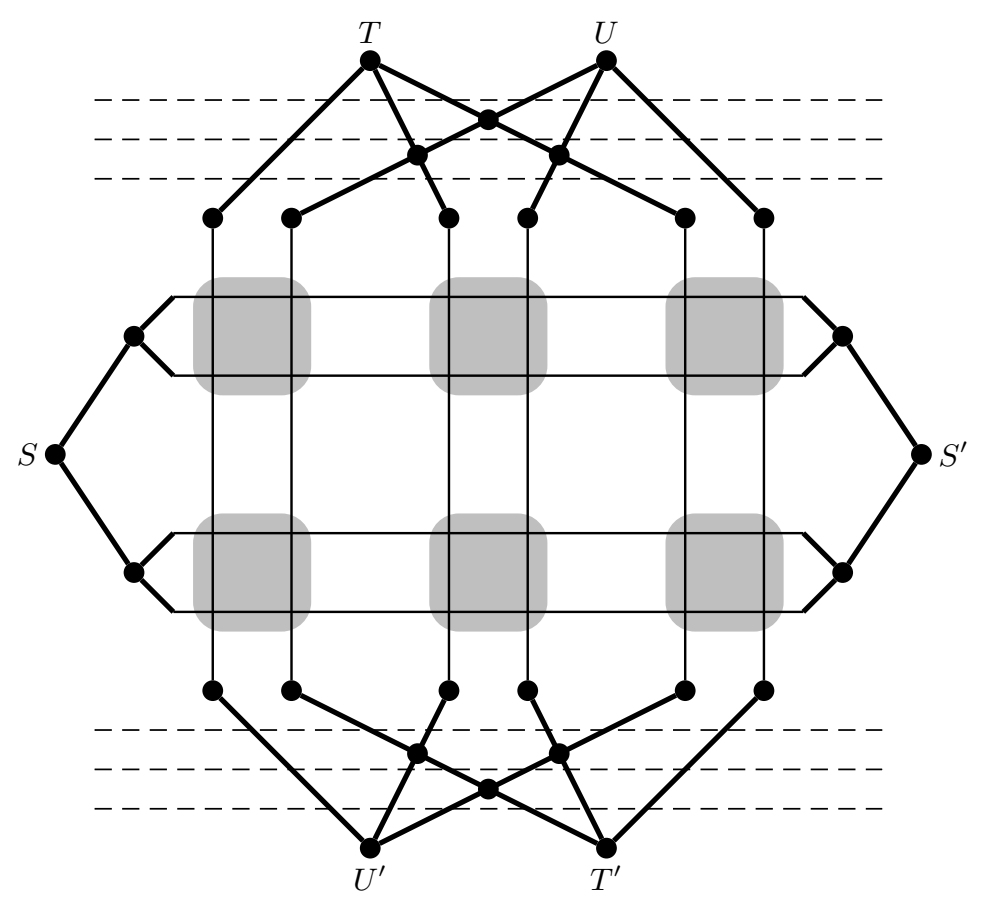

Fig. 1.7. Reduction of the demand graph to only three sets of parallel edges. The central part corresponds to the grid built before, thick edges are the new edges. The demands are equal to the number of variables for the demand edge $\left(X, X^{\prime}\right)$, and to the number of clauses for $\left(U, U^{\prime}\right)$ and $\left(V, V^{\prime}\right)$. Dashed lines define tight cuts.

This result can be further strengthened to two demand edges [22]. Keeping Schwärzler's global idea of the reduction from 3-SAT, the details become much more complicated since the two classes of "vertical demand edges" are decreased to one with a tricky idea whose technical realization is also more complicated.

In Schwärzler's proof vertical paths usually do not switch columns (allowing then two horizontal paths per row to cross the column), columns are switched only in one row, where the corresponding variable is set to TRUE in the clause of the column. In this case the corresponding horizontal line is prevented from becoming a path in the multiflow.

The idea now is to do just the opposite in terms of switching columns: vertical paths will usually switch columns, except in the row associated with the particular TRUE valued variable of the column, when they don't switch. The number of rows can be supposed to be even, so in case of a feasible truth assignment there are an odd number of column-switches in every column!

This is realized this time by paths that run in pairs parallelly and never cross. The corresponding demand edges form one parallel class. Let us explain the main ideas of realizing this and the related technical problems of [22]:

First, for convenience, the problem is generalized by forbidding crossing paths in a subset $W \subseteq V$ of vertices of degree 4 . This is not really a generalization, since each $w \in W$ can be split 
into four vertices of degree 1 , with one new vertex for each incident edge; then add a $C_{4}$ between the four new vertices in the cyclic order of the four edges in the planar embedding. Clearly, there is a bijection between the solutions of the edge-disjoint paths problem after the application of these gadgets and the solutions where paths are not crossing in the vertices of $W$ in the original graph. A combination of the two gadgets of Figure 1.8 will be placed in the crossing of columns (associated
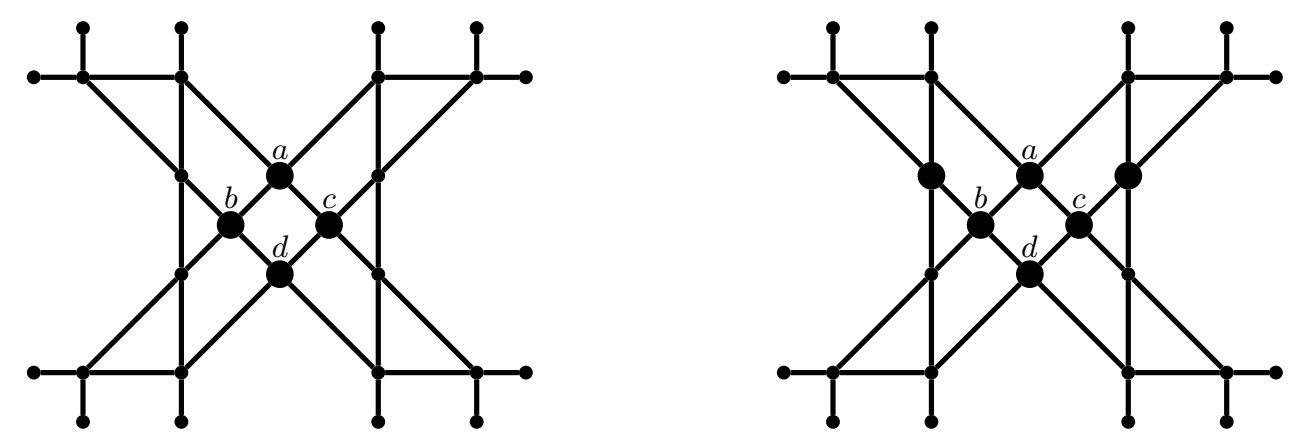

Fig. 1.8. The two gadgets used for the reduction to the case where there are only two demand edges. Two paths can cross in the bold vertices and nowhere else.

with clauses) and rows (associated with variables).

These gadgets have the following properties:

The two parallel vertical paths we mentioned in our general description arrive either from the two left vertices in the upper left corner, or from the two vertices in the upper right corner. Either both stay in the same (left or right) side, or both change sides (column-switch) when they go through the gadget.

If one horizontal path goes through the left gadget, then the two parallel vertical paths are obliged to switch sides, while in the right gadget they are allowed to stay on the same side. If two horizontal paths go through the gadgets both paths are obliged to switch sides.

A combination of these gadgets placed in the same "grid" as before encodes a truth assignment satisfying the goal we have described. Again, the most difficult part of the proof is to ensure that the paths cannot deviate from their intended itineraries. There are indeed only two kinds of demand edges: vertical and horizontal, as required.

\subsubsection{Acyclic Digraphs}

Schwärzler gave also a directed acyclic version of his reduction [34]:

Theorem 7 (Schwärzler) The arc-disjoint paths problem is NP-complete, even if $G$ is planar and acyclic, and all terminals lie on the outer boundary of $G$.

The trick presented in Subsection 1.4.1 serves now again to reduce the number of terminals:

Theorem 8 The arc-disjoint paths problem is NP-complete, even if $G$ is planar and acyclic, $|E(H)|=3$, and all terminals lie on the outer boundary of $G$.

Both the arc-disjoint and the vertex-disjoint paths problems are polynomial-time solvable when the total number of demand is fixed. We show that the complexity of the vertex-disjoint version is again the same as the edge-disjoint versions when $|E(H)|$ is not bounded, both problems are NP-complete:

Theorem 9 The vertex-disjoint paths problem is NP-complete in acyclic digraphs, even if $G+H$ is planar. 

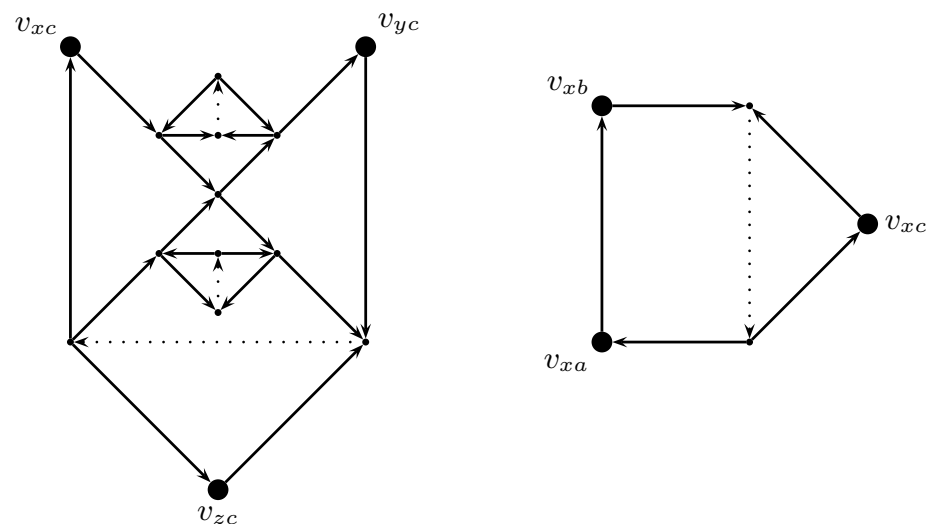

Fig. 1.9. The gadget for the clauses is on the left, for the variables it is on the right. Dotted edges are demand edges, bold vertices are those that subdivide the edges in the original graph.

Proof. The proof is the directed acyclic version of Middendorf and Pfeiffer's proof [19] of their Theorem 1 establishing the NP-hardness of the edge-disjoint paths problem if $G+H$ is planar. (However, again, we cannot reduce the theorem to their result.)

We reduce Planar 3-Sat to the stated problem: let $\varphi$ be a formula whose associated graph is planar, and suppose (without loss of generality) that each variable appears at most three times, exactly once negatively, and there is no clause with twice the same variable. Define the undirected bipartite graph $(C, V, F)$ with the set of clauses $C$ and the set $V$ of variables as classes, and $F:=\{x c$ : variable $x$ appears in clause $c\}$ and subdivide each edge $(x, c)$ into two edges by adding a new node $v_{x c}$.

Take now an arbitrary ordering of the set of variables, and define for each clause a gadget in the following way: choose $z$ to be an arbitrary of the three variables of the clause, and then choose the notation $x$ and $y$ so that $x<y$. With this notation construct the gadget on the left of Figure 1.9 upon the vertices $v_{x c}, v_{y c}, v_{z c}$, adding the other vertices of the figure anew for each clause. Finally delete the vertex representing $c$.

Now for each variable vertex $x$ occurring in three clauses, let $a$ and $b$ be the clauses in which $x$ occurs positively (in arbitrary order), and $c$ the one in which it is negated, and put the gadget depicted in the right side of Figure 1.9 upon the vertices $v_{x a}, v_{x b}, v_{x c}$. (If $x$ occurs only twice, positively in $a$ and negatively in $c$, we add the vertex $v_{x b}$ artificially.) Let $G_{\varphi}, H_{\varphi}$ denote the constructed graph and the constructed demand graph.

Then we have to prove that there exist arc-disjoint paths in $\left(G_{\varphi}, H_{\varphi}\right)$ if and only if $\phi$ is satisfiable. The proof is similar to that of [19], let us sketch it:

It is easy to see that the demand arc in a variable gadget is satisfied either by a path that contains $v_{x c}$ corresponding to $x=T R U E$, or a path that contains the arc $v_{x a} v_{x b}$, which corresponds to $x=F A L S E$.

The demands of a clause gadget can be satisfied if and only if at least one of the three bold vertices of the figure is not used by variable demands, encoding that the clause is satisfied by the variable assignment.

Finally we prove that the digraph is acyclic. Each gadget is acyclic, thus if there is a cycle, it uses at least two gadgets. Suppose for a contradiction that $Q$ is a cycle. Then it intersects clause gadgets in $\left(v_{x a}, v_{y a}\right)$-paths and variable gadgets in $\left(v_{x a}, v_{x b}\right)$-paths. The cycle $Q$ would then follow a sequence where the variable gadgets belong to variables forming an increasing sequence.

\subsection{Key Assertions}

In this section we state the assertions (theorems or problems) that provide (or would provide) most of the results of the tableau: the "minimal" NP-complete problems, and the "maximal" polynomial ones. Those that allowed filling in most of the tableau, also using the basic reductions of section 1.3; and also those problems that were output by the tableau as missing. Some historical results cited 
in the footnotes of the tableau do not reappear here, because they are subsumed by more recent theorems that do reappear. By giving the main theorems in a "full-text" version, we try to provide the most precise formulation and thus a high credibility for the tableau.

We give the list without comment. We hope it will then be easy to switch between the tableau and this list hence and forth to see the facts and their reasons.

\subsubsection{NP-completeness}

Theorem 10 (Fortune, Hopcroft and Wyllie, 1980) The vertex-disjoint paths problem is NPcomplete, even if $E(H)=2$.

Theorem 11 (Middendorf and Pfeiffer 1993) The edge-disjoint paths problem is NP-complete, even if $G+H$ is planar

Theorem 12 (Vygen 1995) The multiflow problem is NP-complete, even if $G$ is an acyclic digraph, $r+c$ is Eulerian and $|E(H)|=3$.

Note that under the same condition, supposing $r(h)=1$, for all $h \in H$, the problem is solvable in polynomial-time but still non-trivial, see the nice algorithm of Ibaraki and Poljak [8].

Theorem 9 The vertex-disjoint paths problem is NP-complete in acyclic digraphs, even if $G+H$ is planar.

Theorem 13 (Marx 2003) The multiflow problem is NP-complete if $G$ is planar (also if it is a grid) and $G+H$ is Eulerian, both in the undirected and directed acyclic case.

Theorem 14 (Naves 2008) The multiflow problem is NP-complete, even with one of the following restrictions:

(i) $G$ is a planar undirected graph, $H$ has only two edges, both on the infinite face of $G$,

(ii) $G$ is a directed graph, $G+H$ is planar, $H$ has only two terminals.

(iii) $G$ is a directed acyclic digraph, $H$ has only two edges, both on the infinite face of $G$.

\subsubsection{Polynomiality}

Theorem 15 (Frank, 1989) The multiflow problem in Eulerian digraphs with $|E(H)|=2$ is solvable in polynomial-time. The cut condition is sufficient for the existence of a solution.

Theorem 16 (Lucchesi and Younger, 1978) The multiflow problem in directed acyclic graphs with $G+H$ planar is solvable in polynomial-time.

Theorem 17 (Fortune, Hopcroft and Wyllie, 1980) The vertex-disjoint paths problem in directed acyclic graphs with $|E(H)|$ bounded is solvable in polynomial-time.

Theorem 18 (Seymour, 1981) The multiflow problem is solvable in polynomial time in undirected graphs, if $G+H$ is planar (or more generally if it does not have a $K_{5}$ minor) and $r+c$ is Eulerian. The cut condition is then necessary and sufficient for the existence of a solution.

Theorem 19 (Lomonosov, 1985) The multiflow problem in Eulerian undirected graphs with $E(H)$ being the union of two stars, or $K_{4}$ or $C_{5}$, is solvable in polynomial-time. The cut condition is sufficient for the existence of a solution.

Theorem 20 (Robertson and Seymour, 1990) The vertex-disjoint and edge-disjoint paths problems in undirected graphs with $r(E(H))$ bounded are solvable in polynomial-time.

Theorem 21 (Schrijver, 1992) The vertex-disjoint paths problem in planar digraphs with $|E(H)|$ bounded is solvable in polynomial-time.

Theorem 22 (Sebô, 1993) The integer multiflow problem in undirected graphs with $|E(H)|$ bounded is solvable in polynomial-time. 


\subsubsection{Relevant open problems}

Last, we state 5 (in fact 7 ) of the 21 open problems that we find particularly nice or frustrating.

Problem 2 (Round-trip problem, [32] Problem 50) Is the problem of finding a connected Eulerian subgraph of a digraph, containing two pre-given vertices, polynomial-time solvable?

Problem 3 Let $k$ be an integer. What is the complexity of routing $k$ pairs of terminals in a Eulerian digraph if $k$ is fixed? Is this problem easier if $G$ is planar?

Ibaraki and Poljak [8] found a polynomial-time algorithm for arbitrary graphs and $k=3$. As far as we know, this is the only partial result about this question.

Problem 4 Is the integer multiflow problem solvable in polynomial-time when $G$ is a Eulerian directed acyclic graph? And when the demand graph is fixed?

Problem 5 What is the complexity of the undirected multiflow problem if $G$ is planar and $G+H$ (or more generally $r+c$ ) is Eulerian and $/ E(H) /$ is fixed?

The complexity of this last problem is open already if $|E(H)|=3$. One of the latest results [18] establishes NP-completeness in both the undirected and directed case, if the number of edges of the demand graph is not fixed. For the directed case this settles Problem 3 if $k$ is not fixed.

\section{References}

1. C. BENTZ, Résolution exacte et approchée de problèmes de multiflot entier et de multicoupe: algorithmes et complexité, thèse de docteur en informatique, Conservatoire Nationale des Arts et Métiers (November 2006).

2. C. BENTZ, M.-C. COSTA, L. LÉTOCART, F. ROUPIN, Minimal multicut and maximal integer multiflow: A survey, EJOR 162 (2005), 55-69.

3. S. EVEN, A. ITAI, A. SHAMIR, On the complexity of Timetable and Multicommodity Flow Problems, SIAM Journal of Computing 5, No.4 (1976), 691-703.

4. S. FORTUNE, J. HOPCROFT, J. WYLLIE, The Directed Subgraph Homeomorphism Problem, Theoretical Computer Science 10 (1980), 111-121.

5. A. FRANK, On connectivity properties of Eulerian digraphs, in Annals of Discrete Mathematics 41, North Holland, Amsterdam, 1989, pp 179-194.

6. A. FRANK, Packing Paths in Planar Graphs, Combinatorica 10 (4) (1990) 325-331.

7. A. FRANK, Packing Paths, Circuits and Cuts - a Survey, in: B. Korte, L. Lovász, H.J. Prőmel, A. Schrijver (Eds.): Paths, Flows, and VLSI-Layout, Springer, Berlin, (1990).

8. T. IBARAKI, S. POLJAK, Weak Three-Linking in Eulerian Digraphs, SIAM Journal on Discrete Mathematics 4 (1991), 84-98.

9. R.M. KARP, On the Complexity of Combinatorial Problems, Networks 5 (1975), 45-68.

10. B. KORTE, L. LOVÁSZ, H.-J. PRÖMEL, A. SCHRIJVER (Eds.), Paths, Flows, and VLSI-Layout, Springer, Berlin, (1990).

11. B. KORTE, J. VYGEN, Combinatorial Optimization: Theory and Algorithms, Algorithms and Combinatorics 21 Springer, Berlin Heidelberg New York, (2000).

12. M.R. KRAMER, J. VAN LEEUWEN, The Complexity of Wire-Routing and Finding the Minimum Area Layouts for Arbitrary VLSI Circuits, in: F.P. Preparata: Advances in Computing Research 2: VLSI Theory, JAI press, London (1984), 129-146.

13. M. LOMONOSOV, Combinatorial Approaches to Multiflow Problems, Discrete Applied Matyhematics 11 (1985), 1-94.

14. M. LOMONOSOV, A. SEBÖ, On the geodesic structure of graphs: a polyhedral approach to metric decomposition, in Integer Programming and Combinatorial Optimization, Proceedings of the 3d IPCO conference, Erice (Italy) 1993, Rinaldi and Wolsey eds. (1993) page 221-234.

15. C.L. LUCCHESI, D.H. YOUNGER, A minimax theorem for directed graphs, The journal of the London Mathematical Society (2) 17 (1978), 369-374.

16. N. LYNCH, The Equivalence of Theorem Proving and the Interconnexion problem, (ACM) SIGDA Newsletters 5:3 (1975), 31-36.

17. L. LOVÁSZ, Combinatorial Problems and Exercises, Akadémiai Kiadó, Budapest, 1979. 
18. D. MARX Eulerian Disjoint paths problem in grid graphs is NP-complete, Discrete Applied Mathematics, 143 (2004) 336-341.

19. M. MIDDENDORF, F. PFEIFFER, On the Complexity of the Disjoint Paths Problem, Combinatorica 13 (1), (1993), 97-107.

20. D. MÜLLER, On the complexity of the planar directed edge-disjoint paths problem, Mathematical Programming, vol. 105, No.2-3 (2006), 275-288.

21. C.St.J.A. NASH-WILLIAMS, in [17] Exercise 6.56, and [32] 71.1a, page 1254.

22. G. NAVES The hardness of routing two pairs on one face, in preparation.

23. H. OKAMURA, P.D. SEYMOUR, Multicommodity flows in planar graphs, Journal of Combinatorial Theory, Series B 31 (1981), 75-81.

24. K. ONAGA, O. KAKUSHO, On Feasibility Conditions of Multicommodity Flows in Networks, IEEE Transactions on Circuit Theory 18 (1971), 425-429

25. P. RAGHAVAN, Ph.D. thesis, Report No. UCB/CSD 87/312, University of California, Berkeley 1986.

26. N. ROBERTSON, P.D. SEYMOUR, Graph Minors XIII. The Disjoint Paths Problem, Journal of Combinatorial Theory B 63 (1995), 65-110.

27. B. ROTHSCHILD, A. WHINSTON, Feasibility of Two-Commodity Network Flows, Operations Research 14 (1966), 1121-1129.

28. A. SCHRIJVER, Applications of Polyhedral Combinatorics to Multicommodity Flows and Compact Surfaces, DIMACS Series in Discrete Mathematics and Theoretical Computer Science, Volume 1, "Polyhedral Combinatorics" eds. W. Cook and P. Seymour, pp. 119-137.

29. A. SCHRIJVER, The Klein Bottle and Multicommodity Flows, Combinatorica 9 (1989), 375-384.

30. A. SCHRIJVER, Homotopic Routing Methods, in: B. Korte, L. Lovász, H.J. Prômel, A. Schrijver (Eds.): Paths, Flows, and VLSI-Layout, Springer, Berlin, (1990).

31. A. SCHRIJVER, Complexity of Disjoint Paths Problems in Planar Graphs, in: T.Lengauer (Ed.): Algorithms - ESA '93, LNCS 726, Springer, Berlin, 1993.

32. A. SCHRIJVER, Combinatorial Optimization: Polyhedra and Efficiency, 1-3, Springer-Verlag Berlin Heidelberg New York, 2003.

33. A. SCHRIJVER, Finding $k$ Disjoint Paths in a Directed Planar Graph, SIAM J. Comput. 23, no.4 (1994), 780-788.

34. W. SCHWÄRZLER, On the complexity of the planar edge-disjoint paths problem with terminals on the outer boundary, accepted in Combinatorica.

35. A. SEBÖ, Integer Plane Multiflows with a Fixed Number of Demands, J. Combinatorial Theory B 59 (1993), 163-171.

36. P.D. SEYMOUR, On Odd Cuts and Planar Multicommodity Flows, Proceedings of the London Mathematical Society III 42 (1981), 178-192.

37. J. VYGEN, NP-Completeness of Some Edge-Disjoint Paths Problems, Discrete Applied Mathematics 61 (1995), 83-90.

38. J. VYGEN, Disjoint Paths, Report No. 94816, Institute for Discrete Mathematics, revised in 1998. 